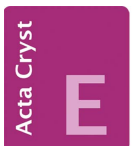

CRYSTALLOGRAPHIC COMMUNICATIONS

ISSN 2056-9890

Received 6 April 2020

Accepted 17 April 2020

Edited by H. Ishida, Okayama University, Japan

Keywords: crystal structure; Michael addition products; cyclohexen-1-one ring; envelope conformation; Hirshfeld surface analysis.

CCDC reference: 1983451

Supporting information: this article has supporting information at journals.iucr.org/e

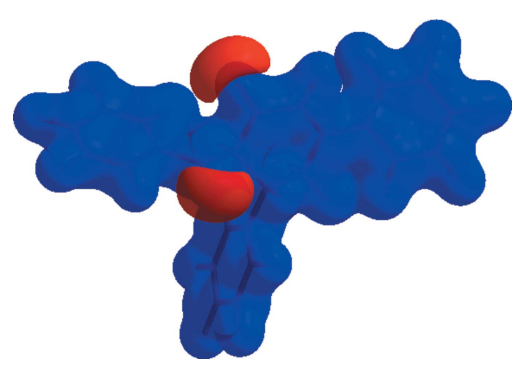

OPEN $\odot$ ACCESS

\section{Crystal structure and Hirshfeld surface analysis of 6-benzoyl-3,5-diphenylcyclohex-2-en-1-one}

\author{
Farid N. Naghiyev, ${ }^{a}$ Mehmet Akkurt, ${ }^{\mathrm{b}}$ Rizvan K. Askerov, ${ }^{a}$ Ibrahim G. Mamedov, \\ Rovnag M. Rzayev, ${ }^{\text {c }}$ Taras Chyrka ${ }^{d}$ and Abel M. Maharramov ${ }^{a}$
}

\begin{abstract}
${ }^{a}$ Organic Chemistry Department, Baku State University, Z. Xalilov str. 23, Az, 1148 Baku, Azerbaijan, ${ }^{\mathbf{b}}$ Department of Physics, Faculty of Sciences, Erciyes University, 38039 Kayseri, Turkey, 'Department of Physics and Chemistry, "Composite Materials" Scientific Research Center, Azerbaijan State Economic University (UNEC), H. Aliyev str. 135, Az, 1063 Baku, Azerbaijan, and dDepartment of Theoretical and Industrial Heat Engineering (TPT), National Technical University of Ukraine, "Igor Sikorsky Kyiv Polytechnic Institute", 03056, Kyiv, Ukraine. *Correspondence e-mail: mustford@ukr.net
\end{abstract}

In the title compound, $\mathrm{C}_{25} \mathrm{H}_{20} \mathrm{O}_{2}$, the central cyclohexenone ring adopts an envelope conformation. The mean plane of the cyclohexenone ring makes dihedral angles of $87.66(11)$ and $23.76(12)^{\circ}$, respectively, with the two attached phenyl rings, while it is inclined by $69.55(11)^{\circ}$ to the phenyl ring of the benzoyl group. In the crystal, the molecules are linked by $\mathrm{C}-\mathrm{H} \cdots \mathrm{O}$ and $\mathrm{C}-\mathrm{H} \cdots \pi$ interactions, forming a three-dimensional network.

\section{Chemical context}

There have been a series of significant examples of enone derivatives used as target products as well as synthetic intermediates (Abdelhamid et al., 2011; Asgarova et al., 2019; Khalilov et al., 2018a,b; Thomas, 2007). Moreover, a number of useful compounds containing enone moieties have been found in nature, such as cyanthiwigin $\mathrm{U},(+)$-cepharamine, phorbol and grandisine $\mathrm{G}$, which were the object of a total synthesis (Pfeiffer et al., 2005; Schultz \& Wang, 1998; Kawamura et al., 2016; Cuthbertson \& Taylor, 2013). As part of a further study on the chemistry of $\alpha, \beta$-unsaturated ketones (Naghiyev et al., 2016), we report herein the crystal structure and Hirshfeld surface analysis of the title compound.

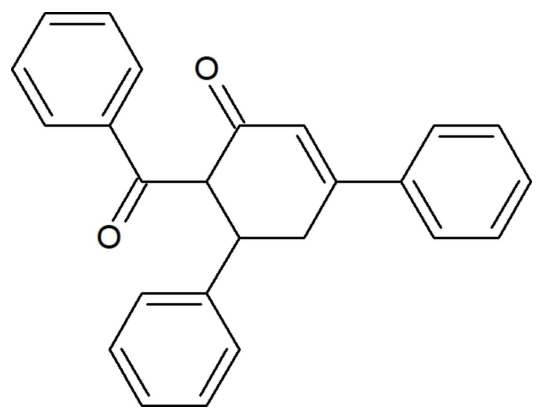

\section{Structural commentary}

In the title compound (Fig. 1), the central cyclohexenone ring adopts an envelope conformation with puckering parameters $Q_{\mathrm{T}}=0.470(2) \AA, \theta=125.3(2)^{\circ}$ and $\varphi=300.8(3)^{\circ}$. The mean plane of the cyclohexenone ring [maximum deviation = $0.335(2) \AA]$ makes dihedral angles of $87.66(11)$ and $23.76(12)^{\circ}$, respectively, with the $\mathrm{C} 14-\mathrm{C} 18$ and $\mathrm{C} 20-\mathrm{C} 25$ 


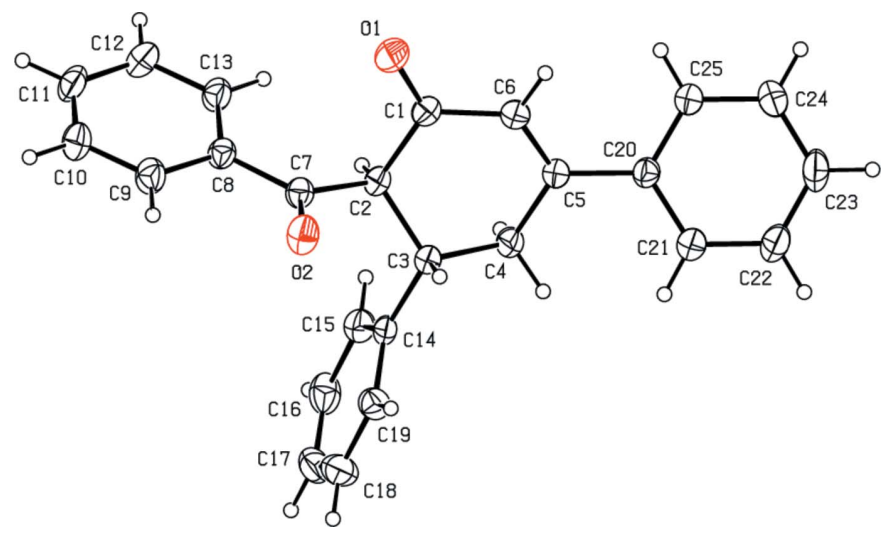

Figure 1

The molecular structure of the title compound, with the atom labelling. Displacement ellipsoids are drawn at the $30 \%$ probability level. $\mathrm{H}$ atoms are shown as spheres of arbitrary radius.

phenyl rings, whereas it is inclined by $69.55(11)^{\circ}$ to the $\mathrm{C} 8-$ C13 phenyl ring of the benzoyl group.

\section{Supramolecular features and Hirshfeld surface analysis}

In the crystal, the molecules are linked by $\mathrm{C}-\mathrm{H} \cdots \mathrm{O}$ and $\mathrm{C}-$ $\mathrm{H} \cdots \pi$ interactions $\left(\mathrm{C} 2-\mathrm{H} 2 A \cdots \mathrm{O} 2^{\mathrm{i}}, \quad \mathrm{C} 15-\mathrm{H} 15 A \cdots \mathrm{O} 1^{\mathrm{i}}\right.$, $\mathrm{C} 22-\mathrm{H} 22 A \cdots \mathrm{O} 1^{\mathrm{ii}}$ and $\mathrm{C} 11-\mathrm{H} 11 A \cdots C g 3^{\mathrm{iii}}$; symmetry codes as given in Table 1; $C g 3$ is the centroid of the C14-C19 ring), forming layers parallel to the $a b$ plane. The layers are further connected by another $\mathrm{C}-\mathrm{H} \cdots \pi$ interaction (C24$\mathrm{H} 24 A \cdots C g 2^{\text {iv }}$; Table $1 ; C g 2$ is the centroid of the $\mathrm{C} 8-\mathrm{C} 13$ ring), forming a three-dimensional network (Fig. 2).

The Hirshfeld surface analysis (Spackman \& Jayatilaka, 2009) was performed using CrystalExplorer 3.1 (Wolff et al., 2012). The surface of the title compound mapped over $d_{\text {norm }}$ is

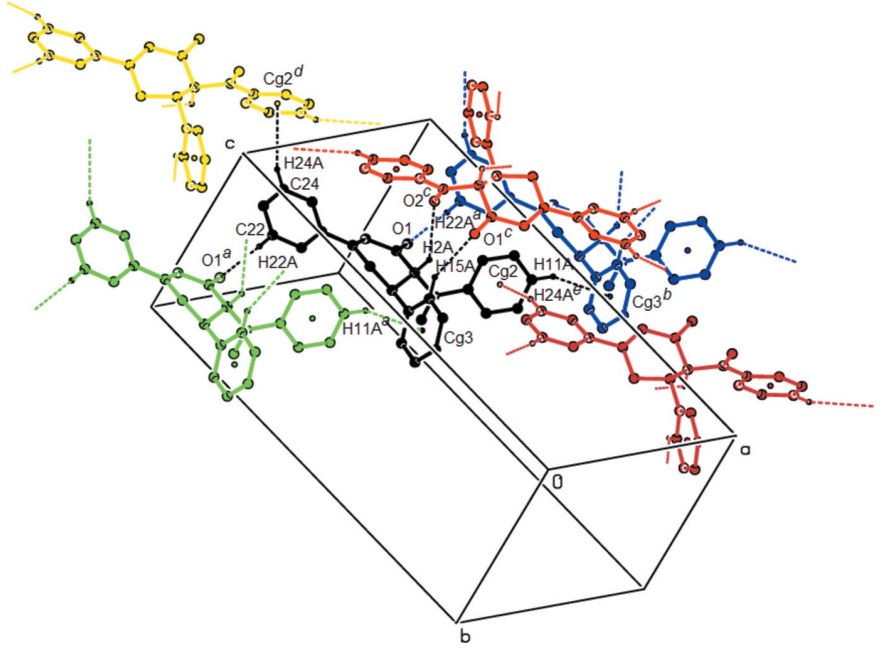

Figure 2

A packing view of the title compound, formed by $\mathrm{C}-\mathrm{H} \cdots \mathrm{O}$ and $\mathrm{C}-$ $\mathrm{H} \cdots \pi$ interactions (dashed lines). [Symmetry codes: (a) $x-1, y, z ;(b)$ $x+1, y, z ;(c)-x+\frac{3}{2}, y-\frac{1}{2},-z+\frac{3}{2} ;(d) x-\frac{1}{2},-y+\frac{1}{2}, z+\frac{1}{2} ;(e) x+\frac{1}{2},-y+\frac{1}{2}$, $\left.z-\frac{1}{2} \cdot\right]$
Table 1

Hydrogen-bond geometry $\left(\AA,^{\circ}\right)$.

$\mathrm{Cg} 2$ and $\mathrm{Cg} 3$ are the centroids of the $\mathrm{C} 8-\mathrm{C} 13$ and $\mathrm{C} 14-\mathrm{C} 19$ phenyl rings, respectively.

\begin{tabular}{lllll}
\hline$D-\mathrm{H} \cdots A$ & $D-\mathrm{H}$ & $\mathrm{H} \cdots A$ & $D \cdots A$ & $D-\mathrm{H} \cdots A$ \\
\hline $\mathrm{C} 2-\mathrm{H} 2 A \cdots \mathrm{O} 2^{\mathrm{i}}$ & 0.98 & 2.50 & $3.251(3)$ & 133 \\
$\mathrm{C} 15-\mathrm{H} 15 A \cdots \mathrm{O} 1^{\mathrm{i}}$ & 0.93 & 2.55 & $3.369(3)$ & 148 \\
$\mathrm{C} 22-\mathrm{H} 22 A \cdots \mathrm{O} 1^{\mathrm{ii}}$ & 0.93 & 2.54 & $3.472(3)$ & 175 \\
$\mathrm{C} 11-\mathrm{H} 11 A \cdots C g 3^{\text {iii }}$ & 0.93 & 2.88 & $3.717(2)$ & 150 \\
$\mathrm{C} 24-\mathrm{H} 24 A \cdots C g 2^{\text {iv }}$ & 0.93 & 2.78 & $3.667(3)$ & 159 \\
\hline
\end{tabular}

Symmetry codes: (i) $-x+\frac{3}{2}, y-\frac{1}{2},-z+\frac{3}{2}$; (ii) $x-1, y, z$; (iii) $x+1, y, z$; (iv) $x-\frac{3}{2},-y-\frac{1}{2}, z-\frac{1}{2}$.

shown in Fig. 3. The dark-red spots on the $d_{\text {norm }}$ surface arise as a result of short interatomic contacts, while the other weaker intermolecular interactions appear as light-red spots. The Hirshfeld surface mapped over electrostatic potential (Spackman et al., 2008; Jayatilaka et al., 2005) is shown in Fig. 4. The blue regions indicate positive electrostatic potential (hydrogen-bond donors), while the red regions indicate negative electrostatic potential (hydrogen-bond acceptors). The overall two-dimensional fingerprint plot (Spackman \& McKinnon, 2002), and those delineated into $\mathrm{H} \cdot \mathrm{H}$ (48.8\%), $\mathrm{C} \cdot \mathrm{H} / \mathrm{H} \cdots \mathrm{C}(34.9 \%)$ and $\mathrm{O} \cdots \mathrm{H} / \mathrm{H} \cdots \mathrm{O}(15 \%)$ contacts are illustrated in Fig. $5 a-d$, respectively. The most significant intermolecular contribution is from the $\mathrm{H} \cdots \mathrm{H}$ contact $(48.8 \%)$ (Fig. $5 b)$. The other minor contributions to the Hirshfeld surface are by $\mathrm{C} \cdots \mathrm{C}(0.9 \%), \mathrm{O} \cdots \mathrm{C} / \mathrm{C} \cdots \mathrm{O}(0.5 \%)$ and $\mathrm{O} \cdots \mathrm{O}(0.1 \%)$ contacts. The large number of $\mathrm{H} \cdots \mathrm{H}$, $\mathrm{C} \cdots \mathrm{H} / \mathrm{H} \cdots \mathrm{C}$ and $\mathrm{O} \cdots \mathrm{H} / \mathrm{H} \cdots \mathrm{O}$ interactions suggest that van der Waals interactions and hydrogen bonding play the major roles in the crystal packing (Hathwar et al., 2015).

\section{Database survey}

Although a search of the Cambridge Structural Database (CSD, Version 5.41, November 2019; Groom et al., 2016) for 3,5-diphenylcyclohex-2-en-1-one derivatives gave 44 hits, no

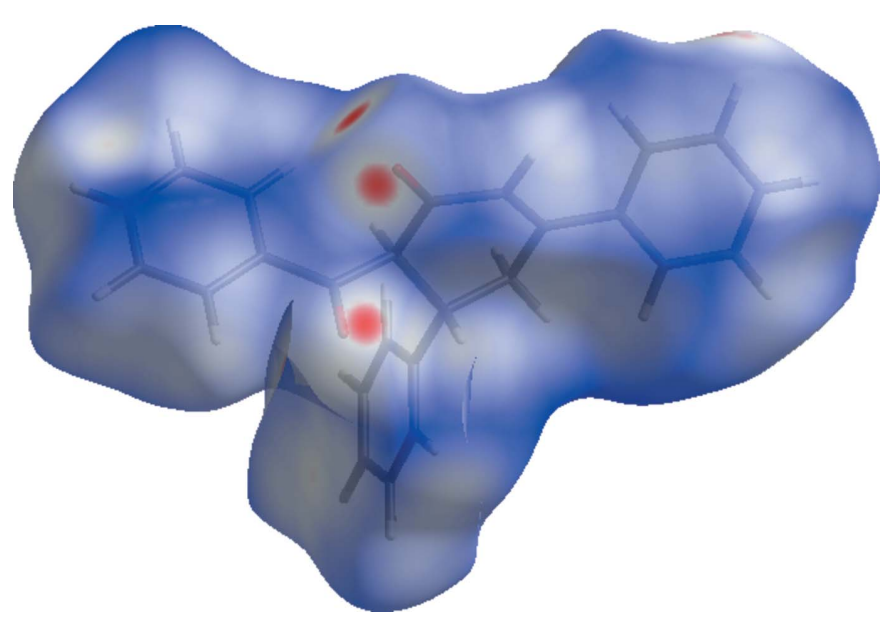

Figure 3

The Hirshfeld surface of the title compound plotted over $d_{\text {norm }}$ using a standard surface resolution with a fixed colour scale of -0.1582 (red) to 1.4399 a.u. (blue). 


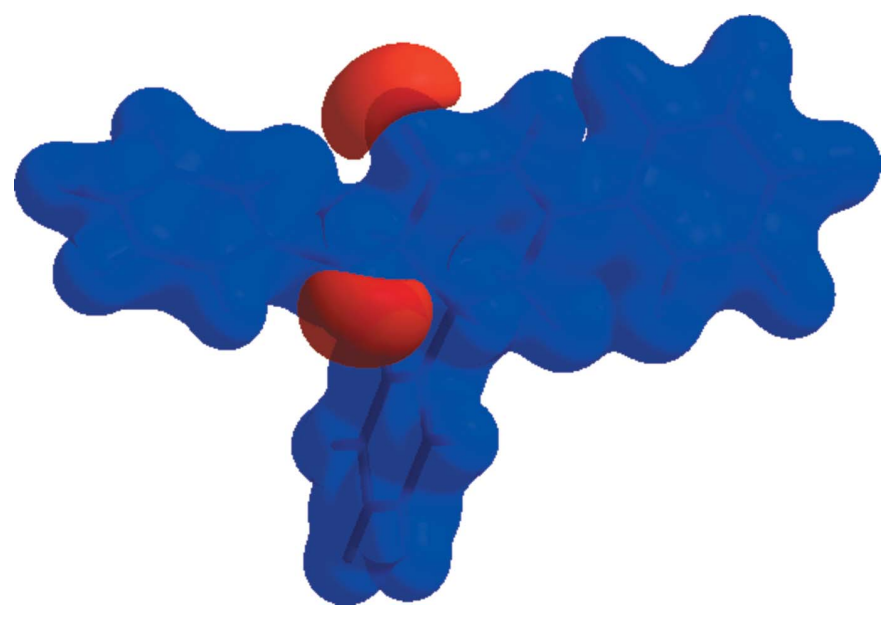

Figure 4

The Hirshfeld surface of the title compound plotted over electrostatic potential energy in the range from -0.0500 to 0.0500 a.u. using the STO$3 \mathrm{G}$ basis set at the Hartree-Fock level of theory. Hydrogen-bond donors and acceptors are shown as blue and red regions around the atoms, corresponding to positive and negative potentials, respectively.

compound having a skeleton of 6-acetyl-3,5-diphenylcyclohex2-en-1-one was found. As related compounds, nine derivatives of ethyl 2-oxo-4,6-diphenylcyclohex-3-ene carboxylate were reported.

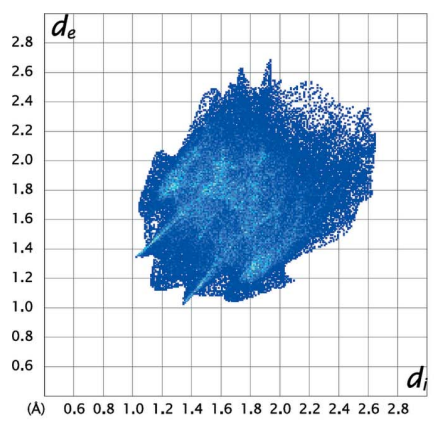

(a) All...All $100 \%$

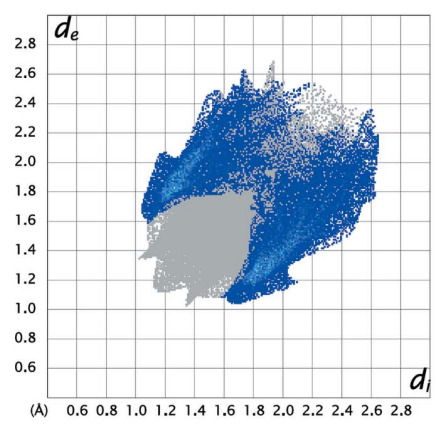

(c) $C \ldots . . H / H \ldots C \quad 34.9 \%$

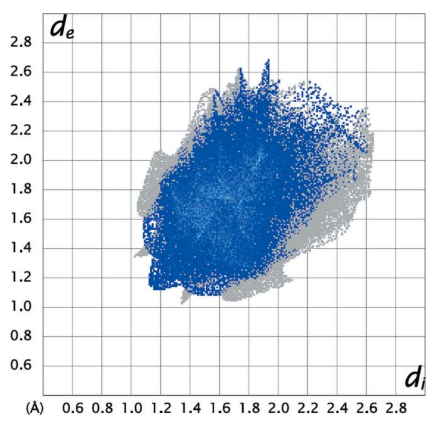

(b) $H \ldots . . H \quad 48.8 \%$

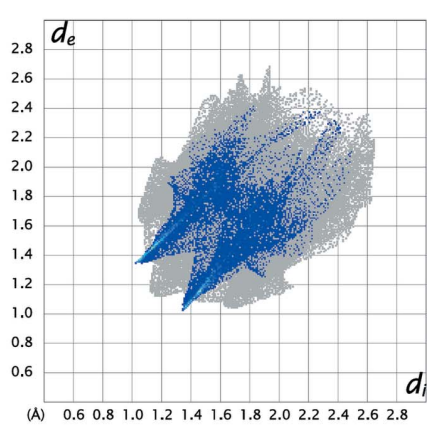

(d) $O \ldots H / H \ldots O \quad 15 \%$
Figure 5

The two-dimensional fingerprint plots for the title compound, showing $(a)$ all interactions, and delineated into $(b) \mathrm{H} \cdots \mathrm{H},(c) \mathrm{C} \cdots \mathrm{H} / \mathrm{H} \cdots \mathrm{C},(d)$ $\mathrm{O} \cdots \mathrm{H} / \mathrm{H} \cdots \mathrm{O}$ interactions. The $d_{\mathrm{i}}$ and $d_{\mathrm{e}}$ values are the closest internal and external distances $(\AA)$ from given points on the Hirshfeld surface.

Table 2

Experimental details.

Crystal data

Chemical formula

$M_{\text {r }}$

Crystal system, space group

Temperature (K)

$a, b, c(\AA)$

$\beta\left({ }^{\circ}\right)$

$V\left(\AA^{3}\right)$

$Z$

Radiation type

$\mu\left(\mathrm{mm}^{-1}\right)$

Crystal size (mm)

Data collection

Diffractometer

Absorption correction

$T_{\min }, T_{\max }$

No. of measured, independent and observed $[I>2 \sigma(I)]$ reflections

$R_{\text {int }}$

$(\sin \theta / \lambda)_{\max }\left(\AA^{-1}\right)$

Refinement

$R\left[F^{2}>2 \sigma\left(F^{2}\right)\right], w R\left(F^{2}\right), S$

No. of reflections

No. of parameters

H-atom treatment

$\Delta \rho_{\max }, \Delta \rho_{\min }\left(\mathrm{e} \AA^{-3}\right)$

Computer programs: APEX2 and SAINT (Bruker, 2003), SHELXT (Sheldrick, 2015a) and SHELXL2018 (Sheldrick, 2015b).

\section{Synthesis and crystallization}

To a solution of 1,3-diphenyl-2-propen-1-one $(1.90 \mathrm{mmol})$ in benzene $(15 \mathrm{ml}), 1$-phenylbutane-1,3-dione $(1.90 \mathrm{mmol})$ and $0.05 \mathrm{ml}$ of dry piperidine were added in this order, and the mixture was stirred at room temperature for $24 \mathrm{~h}$. After completion of the reaction (as monitored by TLC), the solvent was removed under reduced pressure, and the residue was washed with hot water. Then, the products were recrystallized from ethanol (yield 72\%, m.p. $446 \mathrm{~K}$ ). IR (KBr): 2926, 2966, 3006 and $3062 v(\mathrm{CH}), 1610,1650$ and $1676 v(\mathrm{C}=\mathrm{O}) \mathrm{cm}^{-1}$; ${ }^{1} \mathrm{H}$ NMR (300.130 MHz, DMSO-d 6 ): $\delta 3.12\left(d d, 2 \mathrm{H}, \mathrm{CH}_{2},{ }^{2} J_{\mathrm{H}-}\right.$ $\left.{ }_{\mathrm{H}}=16.3 \mathrm{~Hz},{ }^{3} J_{\mathrm{H}-\mathrm{H}}=8.2 \mathrm{~Hz}\right), 3.91\left(t, 1 \mathrm{H}, \mathrm{CH},{ }^{3} J_{\mathrm{H}-\mathrm{H}}=12.4 \mathrm{~Hz}\right)$, $5.52\left(d, 1 \mathrm{H}, \mathrm{CH},{ }^{3} J_{\mathrm{H}-\mathrm{H}}=12.4 \mathrm{~Hz}\right), 6.56(\mathrm{~s}, 1 \mathrm{H}, \mathrm{CH}=), 7.1-7.92$ ( $m$, 15Harom, 3Ar); ${ }^{13} \mathrm{C}$ NMR $\left(75.468 \mathrm{MHz}, \mathrm{DMSO}-d_{6}\right): \delta$ 199.4, 197.5, 159.6, 142.7, 138.3, 137.8, 133.7, 130.9, 129.3, 129.1, 128.8, 128.0, 127.2, 126.9, 124.2, 58.2, 43.9, 36.4; MS (ESI): $m / z$ : $353.15[M+\mathrm{H}]^{+}$.

\section{Refinement}

Crystal data, data collection and structure refinement details are summarized in Table 2. All $\mathrm{H}$ atoms were placed at calculated positions using a riding model, with $\mathrm{C}-\mathrm{H}=0.93-$ $0.98 \AA$, and with $U_{\text {iso }}(\mathrm{H})=1.2 U_{\text {eq }}(\mathrm{C})$. Owing to poor agreement between observed and calculated intensities, eighteen

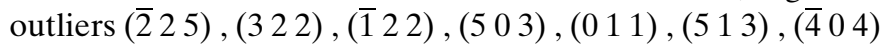
$,(\overline{2} 17),(\overline{5} 23),(\overline{5} 35),(\overline{2} 112),(243),(487),(\overline{3} 07),(\overline{2} 10$ $5),\left(\begin{array}{l}2 \\ 5\end{array} 5\right),\left(\begin{array}{l}\overline{3} 215\end{array}\right)$ and $\left(\begin{array}{lll}0 & 1 & 2\end{array}\right)$ were omitted in the final cycle of refinement. 


\section{References}

Abdelhamid, A. A., Mohamed, S. K., Khalilov, A. N., Gurbanov, A. V. \& Ng, S. W. (2011). Acta Cryst. E67, o2830.

Asgarova, A. R., Khalilov, A. N., Brito, I., Maharramov, A. M., Shikhaliyev, N. G., Cisterna, J., Cárdenas, A., Gurbanov, A. V., Zubkov, F. I. \& Mahmudov, K. T. (2019). Acta Cryst. C75, 342347.

Bruker (2003). APEX2, SAINT and $S A D A B S$. Bruker AXS Inc., Madison, Wisconsin, USA.

Cuthbertson, J. D. \& Taylor, R. J. K. (2013). Angew. Chem. Int. Ed. 52, 1490-1493.

Groom, C. R., Bruno, I. J., Lightfoot, M. P. \& Ward, S. C. (2016). Acta Cryst. B72, 171-179.

Hathwar, V. R., Sist, M., Jørgensen, M. R. V., Mamakhel, A. H., Wang, X., Hoffmann, C. M., Sugimoto, K., Overgaard, J. \& Iversen, B. B. (2015). IUCrJ, 2, 563-574.

Jayatilaka, D., Grimwood, D. J., Lee, A., Lemay, A., Russel, A. J., Taylor, C., Wolff, S. K., Cassam-Chenai, P. \& Whitton, A. (2005). TONTO - A System for Computational Chemistry. Available at: http://hirshfeldsurface.net/

Kawamura, S., Chu, H., Felding, J. \& Baran, P. S. (2016). Nature, 532, 90-93.
Khalilov, A. N., Asgarova, A. R., Gurbanov, A. V., Maharramov, A. M., Nagiyev, F. N. \& Brito, I. (2018a). Z. Kristallogr. New Cryst. Struct. 233, 1019-1020.

Khalilov, A. N., Asgarova, A. R., Gurbanov, A. V., Nagiyev, F. N. \& Brito, I. (2018b). Z. Kristallogr. New Cryst. Struct. 233, 947-948.

Naghiyev, F. N., Gurbanov, A. V., Maharramov, A. M., Mamedov, I. G., Allahverdiyev, M. A. \& Mahmudov, K. T. (2016). J. Iran. Chem. Soc. 13, 1-6.

Pfeiffer, M. W. B. \& Phillips, A. J. (2005). J. Am. Chem. Soc. 127, 5334-5335.

Schultz, A. G. \& Wang, A. (1998). J. Am. Chem. Soc. 120, 8259-8260.

Sheldrick, G. M. (2015a). Acta Cryst. A71, 3-8.

Sheldrick, G. M. (2015b). Acta Cryst. C71, 3-8.

Spackman, M. A. \& McKinnon, J. J. (2002). CrystEngComm, 4, 378392.

Spackman, M. A., McKinnon, J. J. \& Jayatilaka, D. (2008). CrystEngComm, 10, 377-388.

Spackman, M. \& Jayatilaka, D. (2009). CrystEngComm, 11, 19-32.

Thomas, A. W. (2007). Science of Synthesis, Vol. 31a, pp. 337-401. Stuttgart: Thieme.

Wolff, S. K., Grimwood, D. J., McKinnon, J. J., Turner, M. J., Jayatilaka, D. \& Spackman, M. A. (2012). Crystal Explorer. University of Western Australia. 


\section{supporting information}

Acta Cryst. (2020). E76, 720-723 [https://doi.org/10.1107/S2056989020005381]

Crystal structure and Hirshfeld surface analysis of 6-benzoyl-3,5-diphenylcyclohex-2-en-1-one

Farid N. Naghiyev, Mehmet Akkurt, Rizvan K. Askerov, Ibrahim G. Mamedov, Rovnag M.

Rzayev, Taras Chyrka and Abel M. Maharramov

Computing details

Data collection: APEX2 (Bruker, 2003); cell refinement: SAINT (Bruker, 2003); data reduction: SAINT (Bruker, 2003); program(s) used to solve structure: SHELXT (Sheldrick, 2015a); program(s) used to refine structure: SHELXL2018

(Sheldrick, 2015b).

6-Benzoyl-3,5-diphenylcyclohex-2-en-1-one

Crystal data

$\mathrm{C}_{25} \mathrm{H}_{20} \mathrm{O}_{2}$

$M_{r}=352.41$

Monoclinic, $P 2{ }_{1} / n$

$a=10.2365$ (4) $\AA$

$b=9.7989(4) \AA$

$c=19.3759(8) \AA$

$\beta=103.333(2)^{\circ}$

$V=1891.14(13) \AA^{3}$

$Z=4$

$F(000)=744$

$D_{\mathrm{x}}=1.238 \mathrm{Mg} \mathrm{m}^{-3}$

Mo $K \alpha$ radiation, $\lambda=0.71073 \AA$

Cell parameters from 3243 reflections

$\theta=2.5-25.0^{\circ}$

$\mu=0.08 \mathrm{~mm}^{-1}$

$T=296 \mathrm{~K}$

Prism, colourless

$0.23 \times 0.20 \times 0.12 \mathrm{~mm}$

Data collection

Bruker APEXII CCD

4102 independent reflections

diffractometer

$\varphi$ and $\omega$ scans

Absorption correction: multi-scan

(SADABS; Bruker, 2003)

$T_{\min }=0.660, T_{\max }=0.746$

23764 measured reflections

2471 reflections with $I>2 \sigma(I)$

$R_{\text {int }}=0.073$

$\theta_{\max }=27.0^{\circ}, \theta_{\min }=2.1^{\circ}$

$h=-13 \rightarrow 13$

$k=-12 \rightarrow 12$

$l=-24 \rightarrow 24$

\section{Refinement}

Refinement on $F^{2}$

Least-squares matrix: full

$R\left[F^{2}>2 \sigma\left(F^{2}\right)\right]=0.059$

$w R\left(F^{2}\right)=0.149$

$S=1.00$

4102 reflections

244 parameters

0 restraints

Primary atom site location: difference Fourier map

Secondary atom site location: difference Fourier map

Hydrogen site location: inferred from neighbouring sites

$\mathrm{H}$-atom parameters constrained

$w=1 /\left[\sigma^{2}\left(F_{\mathrm{o}}^{2}\right)+(0.0717 P)^{2}+0.020 P\right]$

where $P=\left(F_{\mathrm{o}}{ }^{2}+2 F_{\mathrm{c}}{ }^{2}\right) / 3$

$(\Delta / \sigma)_{\max }<0.001$

$\Delta \rho_{\max }=0.19 \mathrm{e}^{-3}$

$\Delta \rho_{\min }=-0.19$ e $\AA^{-3}$ 


\section{Special details}

Geometry. All esds (except the esd in the dihedral angle between two 1.s. planes) are estimated using the full covariance matrix. The cell esds are taken into account individually in the estimation of esds in distances, angles and torsion angles; correlations between esds in cell parameters are only used when they are defined by crystal symmetry. An approximate (isotropic) treatment of cell esds is used for estimating esds involving l.s. planes.

Fractional atomic coordinates and isotropic or equivalent isotropic displacement parameters $\left(\AA^{2}\right)$

\begin{tabular}{|c|c|c|c|c|}
\hline & $x$ & $y$ & $z$ & $U_{\text {iso }} * / U_{\text {eq }}$ \\
\hline $\mathrm{O} 1$ & $0.84873(15)$ & $0.4571(2)$ & $0.84508(9)$ & $0.0662(5)$ \\
\hline $\mathrm{O} 2$ & $0.73311(16)$ & $0.59881(16)$ & $0.68577(9)$ & $0.0556(4)$ \\
\hline $\mathrm{C} 1$ & $0.7327(2)$ & $0.4250(2)$ & $0.81963(11)$ & $0.0432(5)$ \\
\hline $\mathrm{C} 2$ & $0.68555(19)$ & $0.3941(2)$ & $0.74035(11)$ & $0.0377(5)$ \\
\hline $\mathrm{H} 2 \mathrm{~A}$ & 0.703396 & 0.297799 & 0.732533 & $0.045^{*}$ \\
\hline $\mathrm{C} 3$ & $0.53461(19)$ & $0.4203(2)$ & $0.71377(10)$ & $0.0380(5)$ \\
\hline $\mathrm{H} 3 \mathrm{~A}$ & 0.519851 & 0.518223 & 0.718969 & $0.046^{*}$ \\
\hline $\mathrm{C} 4$ & $0.4572(2)$ & $0.3451(3)$ & $0.76099(11)$ & $0.0471(6)$ \\
\hline $\mathrm{H} 4 \mathrm{~A}$ & 0.362962 & 0.369151 & 0.746260 & $0.056^{*}$ \\
\hline H4B & 0.464862 & 0.247638 & 0.754221 & $0.056^{*}$ \\
\hline $\mathrm{C} 5$ & $0.5064(2)$ & $0.3775(2)$ & $0.83834(10)$ & $0.0388(5)$ \\
\hline C6 & $0.6339(2)$ & $0.4151(2)$ & $0.86315(11)$ & $0.0442(5)$ \\
\hline H6A & 0.661662 & 0.436354 & 0.911083 & $0.053^{*}$ \\
\hline $\mathrm{C} 7$ & $0.7682(2)$ & $0.4818(2)$ & $0.70074(11)$ & $0.0401(5)$ \\
\hline $\mathrm{C} 8$ & 0.89014 (19) & $0.4261(2)$ & $0.68142(11)$ & $0.0403(5)$ \\
\hline C9 & $0.9499(2)$ & $0.5025(3)$ & $0.63619(12)$ & $0.0532(6)$ \\
\hline H9A & 0.912888 & 0.586024 & 0.619070 & $0.064^{*}$ \\
\hline $\mathrm{C} 10$ & $1.0629(2)$ & $0.4558(3)$ & 0.61664 (13) & $0.0612(7)$ \\
\hline H10A & 1.101409 & 0.507482 & 0.586302 & $0.073^{*}$ \\
\hline C11 & $1.1189(2)$ & $0.3329(3)$ & $0.64180(13)$ & $0.0580(7)$ \\
\hline H11A & 1.194874 & 0.301126 & 0.628243 & $0.070^{*}$ \\
\hline $\mathrm{C} 12$ & $1.0623(2)$ & $0.2568(3)$ & $0.68717(13)$ & $0.0570(6)$ \\
\hline $\mathrm{H} 12 \mathrm{~A}$ & 1.101121 & 0.174324 & 0.704782 & $0.068^{*}$ \\
\hline $\mathrm{C} 13$ & $0.9480(2)$ & $0.3026(2)$ & $0.70664(12)$ & $0.0486(6)$ \\
\hline H13A & 0.909822 & 0.250122 & 0.736835 & $0.058^{*}$ \\
\hline C14 & $0.47921(18)$ & $0.3846(2)$ & $0.63661(10)$ & $0.0375(5)$ \\
\hline $\mathrm{C} 15$ & $0.4852(2)$ & $0.2530(3)$ & $0.61136(12)$ & $0.0515(6)$ \\
\hline $\mathrm{H} 15 \mathrm{~A}$ & 0.529328 & 0.185343 & 0.641574 & $0.062^{*}$ \\
\hline $\mathrm{C} 16$ & $0.4257(2)$ & $0.2214(3)$ & $0.54103(13)$ & $0.0604(7)$ \\
\hline H16A & 0.429682 & 0.132362 & 0.525007 & $0.073^{*}$ \\
\hline $\mathrm{C} 17$ & $0.3615(2)$ & $0.3190(3)$ & $0.49526(13)$ & $0.0620(7)$ \\
\hline H17A & 0.322732 & 0.297407 & 0.448269 & $0.074^{*}$ \\
\hline $\mathrm{C} 18$ & $0.3552(3)$ & $0.4496(3)$ & $0.51981(13)$ & $0.0625(7)$ \\
\hline $\mathrm{H} 18 \mathrm{~A}$ & 0.311330 & 0.516816 & 0.489171 & $0.075^{*}$ \\
\hline C19 & $0.4131(2)$ & $0.4825(2)$ & $0.58944(12)$ & $0.0491(6)$ \\
\hline H19A & 0.407775 & 0.571668 & 0.604998 & $0.059^{*}$ \\
\hline $\mathrm{C} 20$ & $0.4106(2)$ & $0.3634(2)$ & $0.88513(11)$ & $0.0398(5)$ \\
\hline $\mathrm{C} 21$ & $0.2742(2)$ & $0.3877(3)$ & $0.86040(13)$ & $0.0550(6)$ \\
\hline $\mathrm{H} 21 \mathrm{~A}$ & 0.241528 & 0.412364 & 0.813221 & $0.066^{*}$ \\
\hline
\end{tabular}


supporting information

\begin{tabular}{lllll}
$\mathrm{C} 22$ & $0.1862(2)$ & $0.3760(3)$ & $0.90421(15)$ & $0.0667(8)$ \\
$\mathrm{H} 22 \mathrm{~A}$ & 0.095277 & 0.393434 & 0.886588 & $0.080^{*}$ \\
$\mathrm{C} 23$ & $0.2324(3)$ & $0.3389(3)$ & $0.97356(15)$ & $0.0662(7)$ \\
$\mathrm{H} 23 \mathrm{~A}$ & 0.173169 & 0.331066 & 1.003253 & $0.079^{*}$ \\
$\mathrm{C} 24$ & $0.3663(3)$ & $0.3132(3)$ & $0.99904(14)$ & $0.0625(7)$ \\
$\mathrm{H} 24 \mathrm{~A}$ & 0.397718 & 0.287297 & 1.046135 & $0.075^{*}$ \\
$\mathrm{C} 25$ & $0.4553(2)$ & $0.3253(3)$ & $0.95566(12)$ & $0.0516(6)$ \\
$\mathrm{H} 25 \mathrm{~A}$ & 0.545976 & 0.307812 & 0.973826 & $0.062^{*}$ \\
\hline
\end{tabular}

Atomic displacement parameters $\left(\AA^{2}\right)$

\begin{tabular}{lllllll}
\hline & $U^{11}$ & $U^{22}$ & $U^{33}$ & $U^{12}$ & $U^{13}$ & $U^{23}$ \\
\hline O1 & $0.0359(9)$ & $0.1016(15)$ & $0.0586(10)$ & $-0.0170(9)$ & $0.0056(8)$ & $-0.0036(9)$ \\
O2 & $0.0606(10)$ & $0.0407(10)$ & $0.0728(11)$ & $0.0030(8)$ & $0.0305(9)$ & $0.0069(8)$ \\
C1 & $0.0342(11)$ & $0.0494(13)$ & $0.0456(13)$ & $-0.0024(10)$ & $0.0084(9)$ & $0.0009(10)$ \\
C2 & $0.0310(10)$ & $0.0410(12)$ & $0.0426(12)$ & $-0.0015(9)$ & $0.0115(9)$ & $0.0000(9)$ \\
C3 & $0.0340(10)$ & $0.0434(12)$ & $0.0379(11)$ & $0.0018(9)$ & $0.0107(9)$ & $-0.0001(9)$ \\
C4 & $0.0342(11)$ & $0.0680(16)$ & $0.0398(12)$ & $-0.0073(10)$ & $0.0101(9)$ & $0.0004(11)$ \\
C5 & $0.0367(11)$ & $0.0434(13)$ & $0.0376(12)$ & $0.0024(9)$ & $0.0112(9)$ & $0.0001(9)$ \\
C6 & $0.0398(12)$ & $0.0545(14)$ & $0.0378(12)$ & $0.0003(10)$ & $0.0074(9)$ & $-0.0019(10)$ \\
C7 & $0.0383(11)$ & $0.0404(13)$ & $0.0428(12)$ & $-0.0034(10)$ & $0.0118(9)$ & $-0.0003(9)$ \\
C8 & $0.0354(11)$ & $0.0432(12)$ & $0.0434(12)$ & $-0.0054(9)$ & $0.0111(9)$ & $-0.0038(10)$ \\
C9 & $0.0485(13)$ & $0.0566(15)$ & $0.0587(15)$ & $-0.0081(11)$ & $0.0211(11)$ & $0.0045(12)$ \\
C10 & $0.0459(14)$ & $0.0793(19)$ & $0.0643(17)$ & $-0.0090(13)$ & $0.0248(12)$ & $0.0062(14)$ \\
C11 & $0.0313(11)$ & $0.082(2)$ & $0.0644(16)$ & $-0.0024(12)$ & $0.0176(11)$ & $-0.0128(14)$ \\
C12 & $0.0389(12)$ & $0.0618(16)$ & $0.0717(16)$ & $0.0052(11)$ & $0.0159(12)$ & $0.0005(13)$ \\
C13 & $0.0407(12)$ & $0.0528(15)$ & $0.0557(14)$ & $-0.0018(10)$ & $0.0183(10)$ & $0.0054(11)$ \\
C14 & $0.0298(10)$ & $0.0491(13)$ & $0.0365(11)$ & $-0.0019(9)$ & $0.0136(8)$ & $-0.0015(9)$ \\
C15 & $0.0490(13)$ & $0.0568(15)$ & $0.0501(14)$ & $0.0037(11)$ & $0.0141(11)$ & $-0.0028(12)$ \\
C16 & $0.0615(16)$ & $0.0654(17)$ & $0.0603(16)$ & $-0.0112(13)$ & $0.0261(13)$ & $-0.0215(13)$ \\
C17 & $0.0562(15)$ & $0.092(2)$ & $0.0387(13)$ & $-0.0195(15)$ & $0.0123(11)$ & $-0.0051(14)$ \\
C18 & $0.0600(16)$ & $0.0763(19)$ & $0.0468(14)$ & $-0.0068(13)$ & $0.0035(12)$ & $0.0123(13)$ \\
C19 & $0.0496(13)$ & $0.0522(14)$ & $0.0455(13)$ & $-0.0026(11)$ & $0.0110(10)$ & $0.0046(11)$ \\
C20 & $0.0369(11)$ & $0.0449(12)$ & $0.0393(12)$ & $0.0016(9)$ & $0.0122(9)$ & $-0.0039(9)$ \\
C21 & $0.0421(12)$ & $0.0775(18)$ & $0.0470(14)$ & $0.0041(12)$ & $0.0137(11)$ & $-0.0002(12)$ \\
C22 & $0.0405(13)$ & $0.099(2)$ & $0.0657(18)$ & $0.0000(13)$ & $0.0214(12)$ & $-0.0073(15)$ \\
C23 & $0.0624(16)$ & $0.083(2)$ & $0.0653(18)$ & $0.0023(14)$ & $0.0404(14)$ & $-0.0014(14)$ \\
C24 & $0.0645(16)$ & $0.0813(19)$ & $0.0476(14)$ & $0.0096(14)$ & $0.0254(12)$ & $0.0091(13)$ \\
C25 & $0.0429(12)$ & $0.0707(17)$ & $0.0434(13)$ & $0.0104(11)$ & $0.0146(10)$ & $0.0034(11)$ \\
& & & & &
\end{tabular}

Geometric parameters $\left(\AA,{ }^{o}\right)$

\begin{tabular}{llll}
\hline $\mathrm{O} 1-\mathrm{C} 1$ & $1.218(2)$ & $\mathrm{C} 12-\mathrm{C} 13$ & $1.384(3)$ \\
$\mathrm{O} 2-\mathrm{C} 7$ & $1.217(2)$ & $\mathrm{C} 12-\mathrm{H} 12 \mathrm{~A}$ & 0.9300 \\
$\mathrm{C} 1-\mathrm{C} 6$ & $1.461(3)$ & $\mathrm{C} 13-\mathrm{H} 13 \mathrm{~A}$ & 0.9300 \\
$\mathrm{C} 1-\mathrm{C} 2$ & $1.530(3)$ & $\mathrm{C} 14-\mathrm{C} 15$ & $1.385(3)$ \\
$\mathrm{C} 2-\mathrm{C} 7$ & $1.530(3)$ & $\mathrm{C} 14-\mathrm{C} 19$ & $1.389(3)$ \\
$\mathrm{C} 2-\mathrm{C} 3$ & $1.533(3)$ & $\mathrm{C} 15-\mathrm{C} 16$ & $1.393(3)$
\end{tabular}




\begin{tabular}{|c|c|c|c|}
\hline $\mathrm{C} 2-\mathrm{H} 2 \mathrm{~A}$ & 0.9800 & $\mathrm{C} 15-\mathrm{H} 15 \mathrm{~A}$ & 0.9300 \\
\hline $\mathrm{C} 3-\mathrm{C} 14$ & $1.513(3)$ & $\mathrm{C} 16-\mathrm{C} 17$ & $1.366(4)$ \\
\hline $\mathrm{C} 3-\mathrm{C} 4$ & $1.530(3)$ & $\mathrm{C} 16-\mathrm{H} 16 \mathrm{~A}$ & 0.9300 \\
\hline $\mathrm{C} 3-\mathrm{H} 3 \mathrm{~A}$ & 0.9800 & $\mathrm{C} 17-\mathrm{C} 18$ & $1.371(4)$ \\
\hline $\mathrm{C} 4-\mathrm{C} 5$ & $1.501(3)$ & C17-H17A & 0.9300 \\
\hline $\mathrm{C} 4-\mathrm{H} 4 \mathrm{~A}$ & 0.9700 & $\mathrm{C} 18-\mathrm{C} 19$ & $1.381(3)$ \\
\hline $\mathrm{C} 4-\mathrm{H} 4 \mathrm{~B}$ & 0.9700 & $\mathrm{C} 18-\mathrm{H} 18 \mathrm{~A}$ & 0.9300 \\
\hline $\mathrm{C} 5-\mathrm{C} 6$ & $1.335(3)$ & C19-H19A & 0.9300 \\
\hline $\mathrm{C} 5-\mathrm{C} 20$ & $1.487(3)$ & $\mathrm{C} 20-\mathrm{C} 25$ & $1.388(3)$ \\
\hline C6-H6A & 0.9300 & $\mathrm{C} 20-\mathrm{C} 21$ & $1.388(3)$ \\
\hline $\mathrm{C} 7-\mathrm{C} 8$ & $1.487(3)$ & $\mathrm{C} 21-\mathrm{C} 22$ & $1.378(3)$ \\
\hline $\mathrm{C} 8-\mathrm{C} 13$ & $1.387(3)$ & $\mathrm{C} 21-\mathrm{H} 21 \mathrm{~A}$ & 0.9300 \\
\hline $\mathrm{C} 8-\mathrm{C} 9$ & $1.396(3)$ & $\mathrm{C} 22-\mathrm{C} 23$ & $1.367(4)$ \\
\hline $\mathrm{C} 9-\mathrm{C} 10$ & $1.376(3)$ & $\mathrm{C} 22-\mathrm{H} 22 \mathrm{~A}$ & 0.9300 \\
\hline $\mathrm{C} 9-\mathrm{H} 9 \mathrm{~A}$ & 0.9300 & $\mathrm{C} 23-\mathrm{C} 24$ & $1.370(3)$ \\
\hline $\mathrm{C} 10-\mathrm{C} 11$ & $1.374(4)$ & $\mathrm{C} 23-\mathrm{H} 23 \mathrm{~A}$ & 0.9300 \\
\hline $\mathrm{C} 10-\mathrm{H} 10 \mathrm{~A}$ & 0.9300 & $\mathrm{C} 24-\mathrm{C} 25$ & $1.379(3)$ \\
\hline $\mathrm{C} 11-\mathrm{C} 12$ & $1.378(3)$ & $\mathrm{C} 24-\mathrm{H} 24 \mathrm{~A}$ & 0.9300 \\
\hline $\mathrm{C} 11-\mathrm{H} 11 \mathrm{~A}$ & 0.9300 & $\mathrm{C} 25-\mathrm{H} 25 \mathrm{~A}$ & 0.9300 \\
\hline $\mathrm{O} 1-\mathrm{C} 1-\mathrm{C} 6$ & $121.6(2)$ & $\mathrm{C} 11-\mathrm{C} 12-\mathrm{C} 13$ & $120.3(2)$ \\
\hline $\mathrm{O} 1-\mathrm{C} 1-\mathrm{C} 2$ & $120.64(19)$ & $\mathrm{C} 11-\mathrm{C} 12-\mathrm{H} 12 \mathrm{~A}$ & 119.8 \\
\hline $\mathrm{C} 6-\mathrm{C} 1-\mathrm{C} 2$ & $117.80(17)$ & $\mathrm{C} 13-\mathrm{C} 12-\mathrm{H} 12 \mathrm{~A}$ & 119.8 \\
\hline $\mathrm{C} 1-\mathrm{C} 2-\mathrm{C} 7$ & $108.05(16)$ & $\mathrm{C} 12-\mathrm{C} 13-\mathrm{C} 8$ & $120.4(2)$ \\
\hline $\mathrm{C} 1-\mathrm{C} 2-\mathrm{C} 3$ & $111.34(16)$ & $\mathrm{C} 12-\mathrm{C} 13-\mathrm{H} 13 \mathrm{~A}$ & 119.8 \\
\hline $\mathrm{C} 7-\mathrm{C} 2-\mathrm{C} 3$ & $111.64(17)$ & $\mathrm{C} 8-\mathrm{C} 13-\mathrm{H} 13 \mathrm{~A}$ & 119.8 \\
\hline $\mathrm{C} 1-\mathrm{C} 2-\mathrm{H} 2 \mathrm{~A}$ & 108.6 & $\mathrm{C} 15-\mathrm{C} 14-\mathrm{C} 19$ & $117.7(2)$ \\
\hline $\mathrm{C} 7-\mathrm{C} 2-\mathrm{H} 2 \mathrm{~A}$ & 108.6 & $\mathrm{C} 15-\mathrm{C} 14-\mathrm{C} 3$ & $121.83(19)$ \\
\hline $\mathrm{C} 3-\mathrm{C} 2-\mathrm{H} 2 \mathrm{~A}$ & 108.6 & $\mathrm{C} 19-\mathrm{C} 14-\mathrm{C} 3$ & $120.35(19)$ \\
\hline $\mathrm{C} 14-\mathrm{C} 3-\mathrm{C} 4$ & $110.59(16)$ & $\mathrm{C} 14-\mathrm{C} 15-\mathrm{C} 16$ & $120.5(2)$ \\
\hline $\mathrm{C} 14-\mathrm{C} 3-\mathrm{C} 2$ & $114.35(16)$ & $\mathrm{C} 14-\mathrm{C} 15-\mathrm{H} 15 \mathrm{~A}$ & 119.7 \\
\hline $\mathrm{C} 4-\mathrm{C} 3-\mathrm{C} 2$ & $109.83(16)$ & $\mathrm{C} 16-\mathrm{C} 15-\mathrm{H} 15 \mathrm{~A}$ & 119.7 \\
\hline $\mathrm{C} 14-\mathrm{C} 3-\mathrm{H} 3 \mathrm{~A}$ & 107.2 & $\mathrm{C} 17-\mathrm{C} 16-\mathrm{C} 15$ & $121.0(2)$ \\
\hline $\mathrm{C} 4-\mathrm{C} 3-\mathrm{H} 3 \mathrm{~A}$ & 107.2 & $\mathrm{C} 17-\mathrm{C} 16-\mathrm{H} 16 \mathrm{~A}$ & 119.5 \\
\hline $\mathrm{C} 2-\mathrm{C} 3-\mathrm{H} 3 \mathrm{~A}$ & 107.2 & $\mathrm{C} 15-\mathrm{C} 16-\mathrm{H} 16 \mathrm{~A}$ & 119.5 \\
\hline $\mathrm{C} 5-\mathrm{C} 4-\mathrm{C} 3$ & $113.23(17)$ & $\mathrm{C} 16-\mathrm{C} 17-\mathrm{C} 18$ & $118.8(2)$ \\
\hline $\mathrm{C} 5-\mathrm{C} 4-\mathrm{H} 4 \mathrm{~A}$ & 108.9 & $\mathrm{C} 16-\mathrm{C} 17-\mathrm{H} 17 \mathrm{~A}$ & 120.6 \\
\hline $\mathrm{C} 3-\mathrm{C} 4-\mathrm{H} 4 \mathrm{~A}$ & 108.9 & $\mathrm{C} 18-\mathrm{C} 17-\mathrm{H} 17 \mathrm{~A}$ & 120.6 \\
\hline $\mathrm{C} 5-\mathrm{C} 4-\mathrm{H} 4 \mathrm{~B}$ & 108.9 & $\mathrm{C} 17-\mathrm{C} 18-\mathrm{C} 19$ & $120.8(2)$ \\
\hline $\mathrm{C} 3-\mathrm{C} 4-\mathrm{H} 4 \mathrm{~B}$ & 108.9 & $\mathrm{C} 17-\mathrm{C} 18-\mathrm{H} 18 \mathrm{~A}$ & 119.6 \\
\hline $\mathrm{H} 4 \mathrm{~A}-\mathrm{C} 4-\mathrm{H} 4 \mathrm{~B}$ & 107.7 & $\mathrm{C} 19-\mathrm{C} 18-\mathrm{H} 18 \mathrm{~A}$ & 119.6 \\
\hline $\mathrm{C} 6-\mathrm{C} 5-\mathrm{C} 20$ & $122.24(19)$ & $\mathrm{C} 18-\mathrm{C} 19-\mathrm{C} 14$ & $121.1(2)$ \\
\hline $\mathrm{C} 6-\mathrm{C} 5-\mathrm{C} 4$ & $119.54(18)$ & $\mathrm{C} 18-\mathrm{C} 19-\mathrm{H} 19 \mathrm{~A}$ & 119.5 \\
\hline $\mathrm{C} 20-\mathrm{C} 5-\mathrm{C} 4$ & $118.20(17)$ & $\mathrm{C} 14-\mathrm{C} 19-\mathrm{H} 19 \mathrm{~A}$ & 119.5 \\
\hline $\mathrm{C} 5-\mathrm{C} 6-\mathrm{C} 1$ & $124.03(19)$ & $\mathrm{C} 25-\mathrm{C} 20-\mathrm{C} 21$ & $117.5(2)$ \\
\hline $\mathrm{C} 5-\mathrm{C} 6-\mathrm{H} 6 \mathrm{~A}$ & 118.0 & $\mathrm{C} 25-\mathrm{C} 20-\mathrm{C} 5$ & $120.77(18)$ \\
\hline $\mathrm{C} 1-\mathrm{C} 6-\mathrm{H} 6 \mathrm{~A}$ & 118.0 & $\mathrm{C} 21-\mathrm{C} 20-\mathrm{C} 5$ & $121.71(19)$ \\
\hline $\mathrm{O} 2-\mathrm{C} 7-\mathrm{C} 8$ & $120.30(19)$ & $\mathrm{C} 22-\mathrm{C} 21-\mathrm{C} 20$ & $121.5(2)$ \\
\hline
\end{tabular}




\begin{tabular}{|c|c|c|c|}
\hline $\mathrm{O} 2-\mathrm{C} 7-\mathrm{C} 2$ & $118.76(18)$ & $\mathrm{C} 22-\mathrm{C} 21-\mathrm{H} 21 \mathrm{~A}$ & 119.3 \\
\hline $\mathrm{C} 8-\mathrm{C} 7-\mathrm{C} 2$ & $120.94(19)$ & $\mathrm{C} 20-\mathrm{C} 21-\mathrm{H} 21 \mathrm{~A}$ & 119.3 \\
\hline $\mathrm{C} 13-\mathrm{C} 8-\mathrm{C} 9$ & $118.4(2)$ & $\mathrm{C} 23-\mathrm{C} 22-\mathrm{C} 21$ & $120.0(2)$ \\
\hline $\mathrm{C} 13-\mathrm{C} 8-\mathrm{C} 7$ & $123.14(19)$ & $\mathrm{C} 23-\mathrm{C} 22-\mathrm{H} 22 \mathrm{~A}$ & 120.0 \\
\hline $\mathrm{C} 9-\mathrm{C} 8-\mathrm{C} 7$ & $118.4(2)$ & $\mathrm{C} 21-\mathrm{C} 22-\mathrm{H} 22 \mathrm{~A}$ & 120.0 \\
\hline $\mathrm{C} 10-\mathrm{C} 9-\mathrm{C} 8$ & $120.8(2)$ & $\mathrm{C} 22-\mathrm{C} 23-\mathrm{C} 24$ & $119.6(2)$ \\
\hline $\mathrm{C} 10-\mathrm{C} 9-\mathrm{H} 9 \mathrm{~A}$ & 119.6 & $\mathrm{C} 22-\mathrm{C} 23-\mathrm{H} 23 \mathrm{~A}$ & 120.2 \\
\hline $\mathrm{C} 8-\mathrm{C} 9-\mathrm{H} 9 \mathrm{~A}$ & 119.6 & $\mathrm{C} 24-\mathrm{C} 23-\mathrm{H} 23 \mathrm{~A}$ & 120.2 \\
\hline $\mathrm{C} 11-\mathrm{C} 10-\mathrm{C} 9$ & $120.2(2)$ & $\mathrm{C} 23-\mathrm{C} 24-\mathrm{C} 25$ & $120.7(2)$ \\
\hline $\mathrm{C} 11-\mathrm{C} 10-\mathrm{H} 10 \mathrm{~A}$ & 119.9 & $\mathrm{C} 23-\mathrm{C} 24-\mathrm{H} 24 \mathrm{~A}$ & 119.6 \\
\hline $\mathrm{C} 9-\mathrm{C} 10-\mathrm{H} 10 \mathrm{~A}$ & 119.9 & $\mathrm{C} 25-\mathrm{C} 24-\mathrm{H} 24 \mathrm{~A}$ & 119.6 \\
\hline $\mathrm{C} 10-\mathrm{C} 11-\mathrm{C} 12$ & $119.9(2)$ & $\mathrm{C} 24-\mathrm{C} 25-\mathrm{C} 20$ & $120.6(2)$ \\
\hline $\mathrm{C} 10-\mathrm{C} 11-\mathrm{H} 11 \mathrm{~A}$ & 120.1 & $\mathrm{C} 24-\mathrm{C} 25-\mathrm{H} 25 \mathrm{~A}$ & 119.7 \\
\hline $\mathrm{C} 12-\mathrm{C} 11-\mathrm{H} 11 \mathrm{~A}$ & 120.1 & $\mathrm{C} 20-\mathrm{C} 25-\mathrm{H} 25 \mathrm{~A}$ & 119.7 \\
\hline $\mathrm{O} 1-\mathrm{C} 1-\mathrm{C} 2-\mathrm{C} 7$ & $-31.1(3)$ & $\mathrm{C} 10-\mathrm{C} 11-\mathrm{C} 12-\mathrm{C} 13$ & $1.0(4)$ \\
\hline $\mathrm{C} 6-\mathrm{C} 1-\mathrm{C} 2-\mathrm{C} 7$ & $148.64(19)$ & $\mathrm{C} 11-\mathrm{C} 12-\mathrm{C} 13-\mathrm{C} 8$ & $-0.8(4)$ \\
\hline $\mathrm{O} 1-\mathrm{C} 1-\mathrm{C} 2-\mathrm{C} 3$ & $-154.1(2)$ & $\mathrm{C} 9-\mathrm{C} 8-\mathrm{C} 13-\mathrm{C} 12$ & $0.0(3)$ \\
\hline $\mathrm{C} 6-\mathrm{C} 1-\mathrm{C} 2-\mathrm{C} 3$ & $25.7(3)$ & $\mathrm{C} 7-\mathrm{C} 8-\mathrm{C} 13-\mathrm{C} 12$ & $-179.4(2)$ \\
\hline $\mathrm{C} 1-\mathrm{C} 2-\mathrm{C} 3-\mathrm{C} 14$ & $-176.81(17)$ & $\mathrm{C} 4-\mathrm{C} 3-\mathrm{C} 14-\mathrm{C} 15$ & $-63.8(2)$ \\
\hline $\mathrm{C} 7-\mathrm{C} 2-\mathrm{C} 3-\mathrm{C} 14$ & $62.3(2)$ & $\mathrm{C} 2-\mathrm{C} 3-\mathrm{C} 14-\mathrm{C} 15$ & $60.8(3)$ \\
\hline $\mathrm{C} 1-\mathrm{C} 2-\mathrm{C} 3-\mathrm{C} 4$ & $-51.8(2)$ & $\mathrm{C} 4-\mathrm{C} 3-\mathrm{C} 14-\mathrm{C} 19$ & $112.4(2)$ \\
\hline $\mathrm{C} 7-\mathrm{C} 2-\mathrm{C} 3-\mathrm{C} 4$ & $-172.67(17)$ & $\mathrm{C} 2-\mathrm{C} 3-\mathrm{C} 14-\mathrm{C} 19$ & $-123.0(2)$ \\
\hline $\mathrm{C} 14-\mathrm{C} 3-\mathrm{C} 4-\mathrm{C} 5$ & $-179.34(18)$ & $\mathrm{C} 19-\mathrm{C} 14-\mathrm{C} 15-\mathrm{C} 16$ & $-0.4(3)$ \\
\hline $\mathrm{C} 2-\mathrm{C} 3-\mathrm{C} 4-\mathrm{C} 5$ & $53.5(2)$ & $\mathrm{C} 3-\mathrm{C} 14-\mathrm{C} 15-\mathrm{C} 16$ & $175.84(19)$ \\
\hline $\mathrm{C} 3-\mathrm{C} 4-\mathrm{C} 5-\mathrm{C} 6$ & $-27.3(3)$ & $\mathrm{C} 14-\mathrm{C} 15-\mathrm{C} 16-\mathrm{C} 17$ & $0.7(3)$ \\
\hline $\mathrm{C} 3-\mathrm{C} 4-\mathrm{C} 5-\mathrm{C} 20$ & $153.97(19)$ & $\mathrm{C} 15-\mathrm{C} 16-\mathrm{C} 17-\mathrm{C} 18$ & $-0.6(4)$ \\
\hline $\mathrm{C} 20-\mathrm{C} 5-\mathrm{C} 6-\mathrm{C} 1$ & $177.5(2)$ & $\mathrm{C} 16-\mathrm{C} 17-\mathrm{C} 18-\mathrm{C} 19$ & $0.3(4)$ \\
\hline $\mathrm{C} 4-\mathrm{C} 5-\mathrm{C} 6-\mathrm{C} 1$ & $-1.2(3)$ & $\mathrm{C} 17-\mathrm{C} 18-\mathrm{C} 19-\mathrm{C} 14$ & $-0.1(4)$ \\
\hline $\mathrm{O} 1-\mathrm{C} 1-\mathrm{C} 6-\mathrm{C} 5$ & $-178.4(2)$ & $\mathrm{C} 15-\mathrm{C} 14-\mathrm{C} 19-\mathrm{C} 18$ & $0.1(3)$ \\
\hline $\mathrm{C} 2-\mathrm{C} 1-\mathrm{C} 6-\mathrm{C} 5$ & $1.8(3)$ & $\mathrm{C} 3-\mathrm{C} 14-\mathrm{C} 19-\mathrm{C} 18$ & $-176.2(2)$ \\
\hline $\mathrm{C} 1-\mathrm{C} 2-\mathrm{C} 7-\mathrm{O} 2$ & $-83.9(2)$ & $\mathrm{C} 6-\mathrm{C} 5-\mathrm{C} 20-\mathrm{C} 25$ & $-30.6(3)$ \\
\hline $\mathrm{C} 3-\mathrm{C} 2-\mathrm{C} 7-\mathrm{O} 2$ & $38.9(3)$ & $\mathrm{C} 4-\mathrm{C} 5-\mathrm{C} 20-\mathrm{C} 25$ & $148.1(2)$ \\
\hline $\mathrm{C} 1-\mathrm{C} 2-\mathrm{C} 7-\mathrm{C} 8$ & $95.4(2)$ & $\mathrm{C} 6-\mathrm{C} 5-\mathrm{C} 20-\mathrm{C} 21$ & $149.3(2)$ \\
\hline $\mathrm{C} 3-\mathrm{C} 2-\mathrm{C} 7-\mathrm{C} 8$ & $-141.80(19)$ & $\mathrm{C} 4-\mathrm{C} 5-\mathrm{C} 20-\mathrm{C} 21$ & $-32.0(3)$ \\
\hline $\mathrm{O} 2-\mathrm{C} 7-\mathrm{C} 8-\mathrm{C} 13$ & $169.2(2)$ & $\mathrm{C} 25-\mathrm{C} 20-\mathrm{C} 21-\mathrm{C} 22$ & $0.7(4)$ \\
\hline $\mathrm{C} 2-\mathrm{C} 7-\mathrm{C} 8-\mathrm{C} 13$ & $-10.2(3)$ & $\mathrm{C} 5-\mathrm{C} 20-\mathrm{C} 21-\mathrm{C} 22$ & $-179.2(2)$ \\
\hline $\mathrm{O} 2-\mathrm{C} 7-\mathrm{C} 8-\mathrm{C} 9$ & $-10.3(3)$ & $\mathrm{C} 20-\mathrm{C} 21-\mathrm{C} 22-\mathrm{C} 23$ & $-0.5(4)$ \\
\hline $\mathrm{C} 2-\mathrm{C} 7-\mathrm{C} 8-\mathrm{C} 9$ & $170.39(19)$ & $\mathrm{C} 21-\mathrm{C} 22-\mathrm{C} 23-\mathrm{C} 24$ & $-0.1(4)$ \\
\hline $\mathrm{C} 13-\mathrm{C} 8-\mathrm{C} 9-\mathrm{C} 10$ & $0.5(3)$ & $\mathrm{C} 22-\mathrm{C} 23-\mathrm{C} 24-\mathrm{C} 25$ & $0.4(4)$ \\
\hline $\mathrm{C} 7-\mathrm{C} 8-\mathrm{C} 9-\mathrm{C} 10$ & $180.0(2)$ & $\mathrm{C} 23-\mathrm{C} 24-\mathrm{C} 25-\mathrm{C} 20$ & $-0.2(4)$ \\
\hline $\mathrm{C} 8-\mathrm{C} 9-\mathrm{C} 10-\mathrm{C} 11$ & $-0.3(4)$ & $\mathrm{C} 21-\mathrm{C} 20-\mathrm{C} 25-\mathrm{C} 24$ & $-0.4(4)$ \\
\hline $\mathrm{C} 9-\mathrm{C} 10-\mathrm{C} 11-\mathrm{C} 12$ & $-0.5(4)$ & $\mathrm{C} 5-\mathrm{C} 20-\mathrm{C} 25-\mathrm{C} 24$ & $179.6(2)$ \\
\hline
\end{tabular}


Hydrogen-bond geometry $\left(A,{ }^{\circ}\right)$

$\mathrm{Cg} 2$ and $\mathrm{Cg} 3$ are the centroids of the $\mathrm{C} 8-\mathrm{C} 13$ and $\mathrm{C} 14-\mathrm{C} 19$ phenyl rings, respectively.

\begin{tabular}{lllll}
\hline$D-\mathrm{H} \cdots A$ & $D-\mathrm{H}$ & $\mathrm{H} \cdots A$ & $D \cdots A$ & $D-\mathrm{H} \cdots A$ \\
\hline $\mathrm{C} 2-\mathrm{H} 2 A \cdots \mathrm{O} 2^{\mathrm{i}}$ & 0.98 & 2.50 & $3.251(3)$ & 133 \\
$\mathrm{C} 15-\mathrm{H} 15 A \cdots \mathrm{O} 1^{\mathrm{i}}$ & 0.93 & 2.55 & $3.369(3)$ & 148 \\
$\mathrm{C} 22-\mathrm{H} 22 A \cdots \mathrm{O} 1^{\mathrm{ii}}$ & 0.93 & 2.54 & $3.472(3)$ & 175 \\
$\mathrm{C} 11-\mathrm{H} 11 A \cdots C g 3^{\mathrm{ii}}$ & 0.93 & 2.88 & $3.717(2)$ & 150 \\
$\mathrm{C} 24-\mathrm{H} 24 A \cdots C g 2^{\mathrm{iv}}$ & 0.93 & 2.78 & $3.667(3)$ & 159
\end{tabular}

Symmetry codes: (i) $-x+3 / 2, y-1 / 2,-z+3 / 2$; (ii) $x-1, y, z$; (iii) $x+1, y, z$; (iv) $x-3 / 2,-y-1 / 2, z-1 / 2$. 